\title{
CONFORMAL PSEUDO-SUBRIEMANNIAN FUNDAMENTAL GRADED LIE ALGEBRAS OF SEMISIMPLE TYPE
}

\author{
TOMOAKI YATSUI
}

\begin{abstract}
We introduce the notion of a conformal pseudo-subriemannian fundamental graded Lie algebra of semisimple type. Moreover we give a classification of conformal pseudo-subriemannian fundamental graded Lie algebras of semisimple type and their prolongations.
\end{abstract}

\section{INTRODUCTION AND NOTATION}

This paper is the sequel to the previous one [16]. We first recall the notion of fundamental graded Lie algebras. Moreover we define the notion of conformal pseudo-subriemannian fundamental graded Lie algebras, which is a generalization of conformal subriemannian fundamental graded Lie algebras.

A graded Lie algebra (GLA) $\mathfrak{m}=\bigoplus_{p<0} \mathfrak{g}_{p}$ is called a fundamental graded Lie algebra (FGLA) if it is a finite dimensional graded Lie algebra generated by nonzero subspace $\mathfrak{g}_{-1}$. An FGLA $\mathfrak{m}$ is said to be of the $\mu$-th kind if $\mathfrak{g}_{-\mu} \neq\{0\}$ and $\mathfrak{g}_{p}=\{0\}$ for $p<-\mu$. Let $\mathfrak{m}=\bigoplus_{p<0} \mathfrak{g}_{p}$ be an FGLA over $\mathbb{R}$ such that $\mathfrak{g}_{-2} \neq\{0\}$, and let $[g]$ be the conformal class of a nondegenerate symmetric bilinear form $g$ on $\mathfrak{g}_{-1}$. Then the pair $(\mathfrak{m},[g])$ is called a conformal pseudo-subriemannian FGLA. In particular if $g$ is positive definite, then $(\mathfrak{m},[g])$ is called a conformal subriemannian FGLA. Also if the signature of $g$ has the form $(r, r)$, then $(\mathfrak{m},[g])$ is called a conformal neutral-subriemannian FGLA. Note that if $(\mathfrak{m},[g])$ is a conformal pseudo-subriemannian FGLA, so is $(\mathfrak{m},[-g])$. Given two conformal pseudosubriemannian FGLAs $\left(\mathfrak{m}_{1},\left[g_{1}\right]\right)$ and $\left(\mathfrak{m}_{2},\left[g_{2}\right]\right)$ we say that $\left(\mathfrak{m}_{1},\left[g_{1}\right]\right)$ is isomorphic to $\left(\mathfrak{m}_{2},\left[g_{2}\right]\right)$ if there exists a graded Lie algebra isomorphism $\varphi$ of $\mathfrak{m}_{1}$ onto $\mathfrak{m}_{2}$ such that $\left[\varphi^{*} g_{2}\right]=\left[g_{1}\right]$. Also we say that $\left(\mathfrak{m}_{1},\left[g_{1}\right]\right)$ is equivalent to $\left(\mathfrak{m}_{2},\left[g_{2}\right]\right)$ if $\left(\mathfrak{m}_{1},\left[g_{1}\right]\right)$ is isomorphic to $\left(\mathfrak{m}_{2},\left[g_{2}\right]\right)$ or $\left(\mathfrak{m}_{2},\left[-g_{2}\right]\right)$.

Let $(\mathfrak{m},[g])$ be a conformal pseudo-subriemannian FGLA, and let $\mathfrak{g}_{0}$ be the Lie algebra consisting of all the derivations $D$ of $\mathfrak{m}$ satisfying the following conditions: (1) $D\left(\mathfrak{g}_{p}\right) \subset \mathfrak{g}_{p}$ for all $p<0$; (2) $D \mid \mathfrak{g}_{-1} \in \mathfrak{c o}\left(\mathfrak{g}_{-1}, g\right)$. There exists a GLA $\mathfrak{l}=\bigoplus_{p \in \mathbb{Z}} \mathfrak{l}_{p}$ such that: (i) $\mathfrak{g}_{p}=\mathfrak{l}_{p}$ for $p \leqq 0$; (ii) $\mathfrak{l}=\bigoplus_{p \in \mathbb{Z}} \mathfrak{l}_{p}$ is transitive, i.e., for $X \in \mathfrak{l}_{p}, p \geqq 0$, if $\left[X, \mathfrak{l}_{-1}\right]=\{0\}$, then $X=0$; (iii) $\mathfrak{l}=\bigoplus_{p \in \mathbb{Z}} \mathfrak{l}_{p}$ is maximum among GLAs satisfying conditions (i) and (ii) above, which is called the prolongation of $(\mathfrak{m},[g]$ ) (For more details on the prolongation, see $[\mathbf{1 3}, \S 5])$. Note that the prolongation of $(\mathfrak{m},[g])$ is finite dimensional (Lemma 3.2). Clearly the prolongation of $(\mathfrak{m},[g])$ coincides with that of $(\mathfrak{m},[-g])$.

It is known $([\mathbf{5}],[\mathbf{1 6}])$ that the prolongation $\mathfrak{g}=\bigoplus_{p \in \mathbb{Z}} \mathfrak{g}_{p}$ of a conformal subriemannian FGLA $(\mathfrak{m},[g])$ satisfying the condition $\mathfrak{g}_{1} \neq\{0\}$ is a real rank one simple graded Lie algebra. In contrast, there exists a conformal neutral-subriemannian FGLA $(\mathfrak{m},[g])$ such that the prolongation $\mathfrak{g}=\bigoplus_{p \in \mathbb{Z}} \mathfrak{g}_{p}$ of $(\mathfrak{m},[g])$ is nonsemisimple and such that $\mathfrak{g}_{1} \neq\{0\}$ (cf. Example 5.1). A conformal pseudo-subriemannian FGLA is said to be of semisimple type if the prolongation is semisimple. In this paper we give a classification of conformal pseudo-subriemannian FGLAs of semisimple type and their prolongations (Theorem 5.2). In particular we prove that the prolongation of a conformal pseudo-subriemannian FGLA of semisimple type is simple. Also we give a classification of conformal neutral-subriemannian FGLAs of semisimple type (Corollary 5.1). 


\section{Notation and conventions.}

(1) Blackboard bold is used for the standard systems $\mathbb{Z}$ (the ring of integers), $\mathbb{R}$ (real numbers), $\mathbb{C}$ (complex numbers), $\mathbb{C}^{\prime}$ (split complex numbers), the real division rings $\mathbb{H}$ (Hamilton's quaternions), $\mathbb{H}^{\prime}$ (split quaternions), $\mathbb{O}$ (Cayley's [nonassociative] octonions) and $\mathbb{O}^{\prime}$ (split octonions). We denote by $\mathbb{R}_{>0}$ (resp. $\mathbb{R}_{\geq 0}$ ) the set consisting of all the positive real numbers (resp. non-negative real numbers). For $\mathbb{K}=\mathbb{C}, \mathbb{C}^{\prime}, \mathbb{H}, \mathbb{H}^{\prime}, \mathbb{O}$ or $\mathbb{O}^{\prime}$, we set $\operatorname{Im} \mathbb{K}=\{z \in \mathbb{K}: \operatorname{Re} z=0\}$.

(2) For any real vector space $V$ we denote by $V(\mathbb{C})$ the complexification of $V$.

(3) Let $V$ be a finite dimensional real vector space, and let $g$ be a nondegenerate symmetric bilinear form on $V$. We set

$$
\begin{aligned}
& \mathfrak{s o}(V, g)=\{A \in \mathfrak{g l}(V): A \cdot g=0\}, \\
& \mathfrak{c o}(V, g)=\left\{A \in \mathfrak{g l}(V): A \cdot g=\eta_{A} g \text { for some } \eta_{A} \in \mathbb{R}\right\},
\end{aligned}
$$

where $A \cdot g$ is a symmetric bilinear form on $V$ defined by $(A \cdot g)(x, y)=g(A x, y)+g(x, A y)$ $(x, y \in V)$. We define a linear mapping $g^{b}: V \rightarrow V^{*}$ by $g^{b}(x)(y)=g(x, y)(x, y \in V)$. Since $g$ is non-degenerate, $g^{b}$ is a linear isomorphism. We denote by $g^{\sharp}$ the inverse mapping of $g^{b}$.

(4) For a graded vector space $V=\bigoplus_{p \in \mathbb{Z}} V_{p}$ and $k \in \mathbb{Z}$ we denote subspaces $\bigoplus_{p \leqq k} V_{p}$ and $\bigoplus_{p \geqq k} V_{p}$ by $V_{\leqq k}$ and $V_{\geqq k}$ respectively. Also we denote the subspace $\bigoplus_{p<0} V_{p}$ by $V_{-}$. We call $V_{-}$the negative part of $V$.

(5) For a reductive Lie algebra $\mathfrak{g}$, we denote by $\mathfrak{g}^{s s}$ the semisimple part of $\mathfrak{g}$.

(6) For a GLA $\mathfrak{g}=\bigoplus_{p \in \mathbb{Z}} \mathfrak{g}_{p}$ we denote by $\operatorname{Aut}_{0}(\mathfrak{g})$ the group consisting of all the automorphisms $a$ of $\mathfrak{g}$ such that $a\left(\mathfrak{g}_{p}\right)=\mathfrak{g}_{p}$ for all $p \in \mathbb{Z}$.

\section{Finite dimensional SEmisimple GRAded Lie Algebras}

2.1. Finite dimensional complex semisimple graded Lie algebras. Let $\mathfrak{g}=\bigoplus_{p \in \mathbb{Z}} \mathfrak{g}_{p}$ be a complex semisimple GLA such that the negative part $\mathfrak{g}_{-}$is an FGLA. Let $\mathfrak{h}$ be a Cartan subalgebra of $\mathfrak{g}_{0}$; then $\mathfrak{h}$ is a Cartan subalgebra of $\mathfrak{g}$ such that $E \in \mathfrak{h}$, where $E$ is the characteristic element of $\mathfrak{g}=\bigoplus_{p \in \mathbb{Z}} \mathfrak{g}_{p}$ (i.e., $E$ is an element of $\mathfrak{g}_{0}$ such that $[E, X]=p X$ for $X \in \mathfrak{g}_{p}$ ). Let $\Delta$ be a root system of $(\mathfrak{g}, \mathfrak{h})$. For $\alpha \in \Delta$, we denote by $\mathfrak{g}^{\alpha}$ the root space corresponding to $\alpha$. We associate to any set of roots $Q \subset \Delta$ a subspace

$$
\mathfrak{g}(Q)=\sum_{\alpha \in Q} \mathfrak{g}^{\alpha} \subset \mathfrak{g}
$$

There exists a simple root system $\Pi=\left\{\alpha_{1}, \ldots, \alpha_{l}\right\}$ of $(\mathfrak{g}, \mathfrak{h})$ such that $\mathfrak{g}(\Pi) \subset \underset{p \geqq 0}{\bigoplus} \mathfrak{g}_{p}([\mathbf{1 5}, \mathrm{p} .441])$. Clearly $\alpha_{i}(E)$ is a non-negative integer. Since the negative part $\mathfrak{g}_{-}$is generated by $\mathfrak{g}_{-1}, \alpha_{i}(E)$ is 0 or 1 ([15, Lemma 3.8]). We put $\Delta_{p}=\{\alpha \in \Delta: \alpha(E)=p\}$ and $\Pi_{p}=\Delta_{p} \cap \Pi$; then $\Pi=\Pi_{0} \cup \Pi_{1}$. When $\mathfrak{g}=\bigoplus_{p \in \mathbb{Z}} \mathfrak{g}_{p}$ is a simple graded Lie algebra (SGLA), we enumerate simple roots of $\mathfrak{g}$ as in $[\mathbf{3}]$. Moreover if $\mathfrak{g}$ has the Dynkin diagram of type $X_{l}$, then $\mathfrak{g}=\bigoplus_{p \in \mathbb{Z}} \mathfrak{g}_{p}$ is said to be of type $\left(X_{l}, \Pi_{1}\right)$.

For $\gamma \in \Pi_{1}$, we put

$$
\Delta_{-1}(-\gamma)=\left\{-\gamma+\left(\Delta_{0} \cup\{0\}\right)\right\} \cap \Delta=\left\{\alpha=-\gamma+\beta \in \Delta: \beta \in \Delta_{0} \cup\{0\}\right\} .
$$


Proposition 2.1 ([9, Ch.3, §3.5] and [1, Proposition 7.3]). The decomposition of the $\mathfrak{g}_{0}$-module $\mathfrak{g}_{-1}$ into irreducible submodules is given by

$$
\mathfrak{g}_{-1}=\bigoplus_{\gamma \in \Pi_{1}} \mathfrak{g}\left(\Delta_{-1}(-\gamma)\right)
$$

In particular the $\mathfrak{g}_{0}$-module $\mathfrak{g}_{-1}$ is completely reducible. Moreover $\mathfrak{g}\left(\Delta_{-1}(-\gamma)\right)$ is an irreducible $\mathfrak{g}_{0}$-module with highest weight $-\gamma$.

From [4, Ch.VIII, §7, Propositions 11 and 12] and the table of [3] we obtain the following proposition.

Proposition 2.2. Let $\mathfrak{g}=\bigoplus_{p \in \mathbb{Z}} \mathfrak{g}_{p}$ be a finite dimensional complex SGLA satisfying the following conditions: (i) the negative part $\mathfrak{m}$ is an FGLA; (ii) $\mathfrak{g}_{-2}$ and the semisimple part $\mathfrak{g}_{0}^{\text {ss }}$ of $\mathfrak{g}_{0}$ are both nonzero; (iii) there exists a $\mathfrak{g}_{0}^{\text {ss }}$-invariant nondegenerate symmetric bilinear form $g: \mathfrak{g}_{-1} \times \mathfrak{g}_{-1} \rightarrow \mathbb{C}$.

(1) If the $\mathfrak{g}_{0}$-module $\mathfrak{g}_{-1}$ is irreducible, then $\mathfrak{g}=\bigoplus_{p \in \mathbb{Z}} \mathfrak{g}_{p}$ is of type $\left(C_{l},\left\{\alpha_{2}\right\}\right)(l \geqq 3)$ or $\left(F_{4},\left\{\alpha_{4}\right\}\right)$.

(2) If the $\mathfrak{g}_{0}$-module $\mathfrak{g}_{-1}$ is reducible and if $\mathfrak{g}_{-1}$ is the direct sum of two irreducible $\mathfrak{g}_{0}$-submodules of $\mathfrak{g}_{-1}$ which are totally isotropic with respect to $g$, then $\mathfrak{g}=\bigoplus_{p \in \mathbb{Z}} \mathfrak{g}_{p}$ is of type $\left(A_{l},\left\{\alpha_{1}, \alpha_{l}\right\}\right)$ or $\left(B_{l},\left\{\alpha_{1}, \alpha_{l}\right\}\right)(l \geqq 3)$.

Remark 2.1. Let $\mathfrak{g}=\bigoplus_{p \in \mathbb{Z}} \mathfrak{g}_{p}$ be a complex SGLA of type $\left(A_{2},\left\{\alpha_{1}, \alpha_{2}\right\}\right),\left(B_{2},\left\{\alpha_{1}, \alpha_{2}\right\}\right)$ or $\left(G_{2},\left\{\alpha_{1}, \alpha_{2}\right\}\right)$. Then the semisimple part of $\mathfrak{g}_{0}$ is $\{0\}$. We can easily construct a nondegenerate symmetric bilinear form $g$ on $\mathfrak{g}_{-1}$ satisfying the following condition: for any $A \in \mathfrak{g}_{0}$ there exists a $\lambda_{A} \in \mathbb{C}$ such that

$$
g([A, X], Y)+g(X,[A, Y])=\lambda_{A} g(X, Y) \text { for all } X, Y \in \mathfrak{g}_{-1}
$$

(cf. Examples 4.1, 4.2, 4.4).

2.2. Finite dimensional real semisimple graded Lie algebras. In this subsection we describe gradations of finite dimensional real semisimple GLAs. We first notice the following proposition.

Proposition 2.3 ([15, Proposition 3.3]). The finite dimensional real $S G L A s \mathfrak{g}=\bigoplus_{p \in \mathbb{Z}} \mathfrak{g}_{p}$ fall into the following two distinct classes:

(A) The complex SGLAs, considered as real Lie algebras;

(B) The real form of complex simple Lie algebra so that $\mathfrak{g}(\mathbb{C})=\bigoplus_{p \in \mathbb{Z}} \mathfrak{g}_{p}(\mathbb{C})$ is a complex $S G L A$.

Let $\mathfrak{g}=\bigoplus_{p \in \mathbb{Z}} \mathfrak{g}_{p}$ be a finite dimensional real semisimple GLA such that the negative part $\mathfrak{g}_{-}$is an FGLA. Let $E$ be the characteristic element of $\mathfrak{g}=\bigoplus_{p \in \mathbb{Z}} \mathfrak{g}_{p}$, and $\mathfrak{a}$ a maximal $\mathbb{R}$-diagonalizable commutative subalgebra of $\mathfrak{g}$ containing $E$. Clearly $\mathfrak{a}$ is contained in $\mathfrak{g}_{0}$. There exists a Cartan decomposition $\mathfrak{g}=\mathfrak{k} \oplus \mathfrak{p}$ such that $\mathfrak{a} \subset \mathfrak{p}([\mathbf{9}$, Proposition 4.1]). Let $\mathfrak{h}$ be a Cartan subalgebra of $\mathfrak{g}$ containing $\mathfrak{a}$. The complexification $\mathfrak{h}(\mathbb{C})$ of $\mathfrak{h}$ is a Cartan subalgebra of $\mathfrak{g}(\mathbb{C})$. Let $\Delta$ be the root system of $(\mathfrak{g}(\mathbb{C}), \mathfrak{h}(\mathbb{C}))$. We set

$$
\begin{aligned}
& \Delta_{k}=\{\alpha \in \Delta: \alpha(E)=k\} \quad(k \in \mathbb{Z}), \\
& \Delta^{\bullet}=\{\alpha \in \Delta: \alpha(\mathfrak{a})=\{0\}\}, \quad \Delta^{\circ}=\Delta \backslash \Delta^{\bullet} .
\end{aligned}
$$

Let $\sigma$ be the conjugation of $\mathfrak{g}(\mathbb{C})$ defined by its real form $\mathfrak{g}$. For $\lambda \in \mathfrak{h}(\mathbb{C})^{*}$ we define the element $\lambda^{\sigma} \in \mathfrak{h}(\mathbb{C})^{*}$ by $\lambda^{\sigma}=\overline{\lambda \circ \sigma}$. If $\alpha \in \Delta$, then $\alpha^{\sigma} \in \Delta$. We can choose a simple root system $\Pi$ of $(\mathfrak{g}(\mathbb{C}), \mathfrak{h}(\mathbb{C}))$ such that: (i) the corresponding system of positive roots $\Delta^{+}$satisfies the following 
conditions: $\Delta^{+} \cap \Delta^{\circ}$ is $\sigma$-invariant; (ii) $\mathfrak{g}(\Pi) \subset \mathfrak{g}(\mathbb{C})_{\geqq 0}$. There exists an involutive permutation $\nu$ of the set $\Pi^{\circ}$ such that

$$
\gamma^{\sigma}=\nu(\gamma)+\sum_{\beta \in \Pi \bullet} k_{\beta} \beta \quad\left(\gamma \in \Pi^{\circ}, k_{\beta} \in \mathbb{Z}_{\geqq 0}\right) .
$$

We set $\Pi^{\bullet}=\Delta^{\bullet} \cap \Pi, \Pi^{\circ}=\Delta^{\circ} \cap \Pi$ and $\Pi_{k}=\Delta_{k} \cap \Pi$. We shall identify the vertices of the Dynkin diagram $X_{l}$ with the elements of $\Pi$. The Satake diagram $S_{l}$ is obtained from $X_{l}$ as follows: Firstly we paint the vertices $\alpha \in \Pi^{\bullet}$ (resp. $\alpha \in \Pi^{\circ}$ ) into black (resp. white). Secondly for $\alpha \in \Pi^{\circ}$, if $\alpha^{\sigma} \neq \alpha$, then we connect the pair $\left\{\alpha, \alpha^{\sigma}\right\}$ by a curved arrow. When this is done for all such pairs, we obtain the Satake diagram $S_{l}$.

Let $\mathfrak{g}=\bigoplus_{p \in \mathbb{Z}} \mathfrak{g}_{p}$ be a finite dimensional real semisimple GLA with Satake diagram $S_{l}$, and let $\Delta_{k}$, $\Pi$ and $\Pi_{k}$ be as in the above. Since $\mathfrak{g}_{-}$is an FGLA, $\Pi=\Pi_{0} \cup \Pi_{1}$. Furthermore the following properties hold: (i) $\Pi^{\bullet} \subset \Pi_{0}$; (ii) $\Pi_{1} \subset \Pi^{\circ}$; (iii) If $\alpha \in \Pi_{1}$, then $\alpha^{\sigma} \in \Delta_{1}$ ([1, Theorem 8.1]). The semisimple GLA $\mathfrak{g}=\bigoplus_{p \in \mathbb{Z}} \mathfrak{g}_{p}$ is said to be of type $\left(S_{l}, \Pi_{1}\right)([\mathbf{7}, \S 2]$ and $[\mathbf{1 5}, \S 3.4])$. For simplicity we denote by $\mathfrak{g}_{-1}^{\mathbb{C}}(-\gamma)$ the subspace $\mathfrak{g}(\mathbb{C})\left(\Delta_{-1}(-\gamma)\right)$ of $\mathfrak{g}_{-1}(\mathbb{C})$, where $\gamma \in \Pi_{1}$.

Proposition 2.4 ([1, Proposition 8.3]). Let $\mathfrak{g}=\bigoplus_{p \in \mathbb{Z}} \mathfrak{g}_{p}$ be a finite dimensional real semisimple GLA of type $\left(S_{l}, \Pi_{1}\right)$. For $\gamma \in \Pi_{1}$, there are two possibilities:

(1) $\nu(\gamma)=\gamma$. Then $-\gamma^{\sigma} \in \Delta_{-1}(-\gamma)$ and the $\mathfrak{g}_{0}(\mathbb{C})$-module $\mathfrak{g}_{-1}^{\mathbb{C}}(-\gamma)$ is $\sigma$-invariant.

(2) $\nu(\gamma) \neq \gamma$. Then $-\gamma^{\sigma} \in \Delta_{-1}(-\nu(\gamma))$ and the two irreducible $\mathfrak{g}_{0}(\mathbb{C})$-modules $\mathfrak{g}_{-1}^{\mathbb{C}}(-\gamma)$ and $\mathfrak{g}_{-1}^{\mathbb{C}}(-\nu(\gamma))$ determine one irreducible $\mathfrak{g}_{0}$-submodule $\mathfrak{g} \cap\left(\mathfrak{g}_{-1}^{\mathbb{C}}(-\gamma)+\mathfrak{g}_{-1}^{\mathbb{C}}(-\nu(\gamma))\right)$ of $\mathfrak{g}_{-1}$.

\section{Conformal pSeudo-Subriemannian fundamental GRAded Lie Algebras}

Let $\mathfrak{m}=\bigoplus_{p<0} \mathfrak{g}_{p}$ be an FGLA of the $\mu$-kind over $\mathbb{R}$, where $\mu \geqq 2$. Let $g_{1}$ and $g_{2}$ be two nondegenerate real symmetric bilinear forms on $\mathfrak{g}_{-1}$. We say that $g_{1}$ is equivalent to $g_{2}$ if there exists an $\eta \in \mathbb{R}_{>0}$ such that $g_{2}=\eta g_{1}$. We denote by $[g]$ the equivalence class of a nondegenerate real symmetric bilinear form $g$ on $\mathfrak{g}_{-1}$, which is called the conformal class of $g$.

Let $g$ be a nondegenerate real symmetric bilinear form on $\mathfrak{g}_{-1}$ with signature $(r, s)$. We call the pair $(\mathfrak{m},[g])$ a conformal pseudo-subriemannian FGLA of type $(r, s)$. In particular, if $s=0$ (resp. $r=s$ ), then $(\mathfrak{m},[g])$ is called a conformal subriemannian FGLA (resp. a conformal neutralsubriemannian FGLA).

Let $(\mathfrak{m},[g])$ be a conformal pseudo-subriemannian FGLA, and let $\mathfrak{g}_{0}$ be the Lie algebra consisting of all the derivations $D$ of $\mathfrak{m}$ satisfying the following conditions (i) and (ii): (i) $D\left(\mathfrak{g}_{p}\right) \subset \mathfrak{g}_{p}$ for all $p<0$; (ii) $D \mid \mathfrak{g}_{-1} \in \mathfrak{c o}\left(\mathfrak{g}_{-1}, g\right)$. Let $\mathfrak{g}=\bigoplus_{p \in \mathbb{Z}} \mathfrak{g}_{p}$ be the prolongation of $\left(\mathfrak{m}, \mathfrak{g}_{0}\right)$ (see $[\mathbf{1 3}, \S 5.2]$ ). We call the transitive GLA $\mathfrak{g}=\bigoplus_{p \in \mathbb{Z}} \mathfrak{g}_{p}$ the prolongation of $(\mathfrak{m},[g])$. If $\mathfrak{g}$ is finite dimensional and semisimple, then $(\mathfrak{m},[g])$ is said to be of semisimple type.

Let $\left(\mathfrak{m}_{1},\left[g_{1}\right]\right)$ and $\left(\mathfrak{m}_{2},\left[g_{2}\right]\right)$ be two conformal pseudo-subriemannian FGLAs. We say that $\left(\mathfrak{m}_{1},\left[g_{1}\right]\right)$ is isomorphic to $\left(\mathfrak{m}_{2},\left[g_{2}\right]\right)$ if there exists a graded Lie algebra isomorphism $\varphi$ of $\mathfrak{m}_{1}$ onto $\mathfrak{m}_{2}$ such that $\left[\varphi^{*} g_{2}\right]=\left[g_{1}\right]$. Also we say that $\left(\mathfrak{m}_{1},\left[g_{1}\right]\right)$ is equivalent to $\left(\mathfrak{m}_{2},\left[g_{2}\right]\right)$ if $\left(\mathfrak{m}_{1},\left[g_{1}\right]\right)$ is isomorphic to $\left(\mathfrak{m}_{2},\left[g_{2}\right]\right)$ or $\left(\mathfrak{m}_{2},\left[-g_{2}\right]\right)$.

The following lemma can be proved by the same methods as in the case of conformal subriemannian FGLAs ([16, Lemma 3.1]).

Lemma 3.1. Let $(\mathfrak{m},[g])$ be a conformal pseudo-subriemannian $F G L A$, and let $\mathfrak{g}=\bigoplus_{p \in \mathbb{Z}} \mathfrak{g}_{p}$ be the prolongation of $(\mathfrak{m},[g])$. Let $\rho_{-1}$ be the representation of $\mathfrak{g}_{0}$ on $\mathfrak{g}_{-1}$ defined by $\rho_{-1}(A)(X)=[A, X]$ $\left(A \in \mathfrak{g}_{0}, X \in \mathfrak{g}_{-1}\right)$. We set $\hat{\mathfrak{g}}_{0}=\left(\rho_{-1}\right)^{-1}\left(\mathfrak{s o}\left(\mathfrak{g}_{-1}, g\right)\right)$. Then 
(1) $\left[\mathfrak{g}_{0}, \mathfrak{g}_{0}\right] \subset \hat{\mathfrak{g}}_{0}$.

(2) Let $E$ be the characteristic element of $\mathfrak{g}=\bigoplus_{p \in \mathbb{Z}} \mathfrak{g}_{p}$. Then $\mathfrak{g}_{0}=\mathbb{R} E \oplus \hat{\mathfrak{g}}_{0}$.

Lemma 3.2. Let $(\mathfrak{m},[g])$ be a conformal pseudo-subriemannian $F G L A$, and let $\mathfrak{g}=\bigoplus_{p \in \mathbb{Z}} \mathfrak{g}_{p}$ be the prolongation of $(\mathfrak{m},[g])$. Then $\mathfrak{g}$ is finite dimensional.

Proof. We first assume that $\operatorname{dim} \mathfrak{g}_{-1} \geqq 3$. We define a subalgebra $\mathfrak{h}_{0}$ of $\mathfrak{g}_{0}$ as follows:

$$
\mathfrak{h}_{0}=\left\{X \in \mathfrak{g}_{0}:\left[X, \mathfrak{g}_{\leq-2}\right]=\{0\}\right\} .
$$

Identifying $\mathfrak{h}_{0}$ with a subalgebra of $\mathfrak{g l}\left(\mathfrak{g}_{-1}\right)$, we see that $\mathfrak{h}_{0} \subset \mathfrak{c o}\left(\mathfrak{g}_{-1}, g\right)$. Since the second algebraic prolongation $\mathfrak{c o}\left(\mathfrak{g}_{-1}, g\right)^{(2)}$ of $\mathfrak{c o}\left(\mathfrak{g}_{-1}, g\right)$ vanishes, we get $\mathfrak{h}_{0}^{(2)}=\{0\}$. From Corollary 1 of Theorem 11.1 in [13], it follows that $\mathfrak{g}$ is finite dimensional. Next we assume that $\operatorname{dim} \mathfrak{g}_{-1}=2$. There exists a basis $\left(e_{1}, e_{2}\right)$ of $\mathfrak{g}_{-1}$ such that $g\left(e_{i}, e_{j}\right)=\varepsilon_{i} \delta_{i j}$ for all $i, j=1,2$, where $\varepsilon_{i} \in\{-1,1\}$. Note that $\left[e_{1}, e_{2}\right] \neq 0$. For $A \in \mathfrak{h}_{0}$, we set ad $A\left(e_{i}\right)=\sum_{k=1}^{2} a_{k i} e_{k}\left(i=1,2 ; a_{k i} \in \mathbb{R}\right)$. Since $g\left(\left[A, e_{i}\right], e_{j}\right)+g\left(e_{1},\left[A, e_{2}\right]\right)=\lambda_{A} g\left(e_{i}, e_{j}\right)$, we see that $2 a_{i i}=\lambda_{A}$ and $a_{j i} \varepsilon_{j}+a_{i j} \varepsilon_{i}=0$. Also since $\left[A,\left[e_{i}, e_{j}\right]\right]=0$, we get $a_{11}+a_{22}=0$, so $\lambda_{A}=0$. Hence $\mathfrak{h}_{0}$ is considered as a subalgebra of $\mathfrak{s o}\left(\mathfrak{g}_{-1}, g\right)$. However since the first algebraic prolongation $\mathfrak{s o}\left(\mathfrak{g}_{-1}, g\right)^{(1)}$ of $\mathfrak{s o}\left(\mathfrak{g}_{-1}, g\right)$ vanishes, we see that $\mathfrak{g}$ is finite dimensional.

Lemma 3.3. Let $(\mathfrak{m},[g])$ be a conformal pseudo-subriemannian $F G L A$, and let $\mathfrak{g}=\bigoplus_{p \in \mathbb{Z}} \mathfrak{g}_{p}$ be the prolongation of $(\mathfrak{m},[g])$. If $\mathfrak{l}=\bigoplus_{p \in \mathbb{Z}} \mathfrak{l}_{p}$ is a transitive semisimple $G L A$ such that $\mathfrak{g}_{p}=\mathfrak{l}_{p}$ for all $p<0$ and $\operatorname{ad}\left(\mathfrak{l}_{0}\right) \mid \mathfrak{g}_{-1} \subset \mathfrak{c o}\left(\mathfrak{g}_{-1}, g\right)$, then $\mathfrak{g}$ coincides with $\mathfrak{l}$.

Proof. Since $\operatorname{ad}\left(\mathfrak{l}_{0}\right) \mid \mathfrak{g}_{-1} \subset \mathfrak{c o}\left(\mathfrak{g}_{-1}, g\right), \mathfrak{l}=\bigoplus_{p \in \mathbb{Z}} \mathfrak{l}_{p}$ is a graded subalgebra of $\mathfrak{g}=\bigoplus_{p \in \mathbb{Z}} \mathfrak{g}_{p}$. Let $\mathfrak{r}$ be the radical of $\mathfrak{g}$; then $\mathfrak{r}$ is a graded ideal of $\mathfrak{g}=\bigoplus_{p \in \mathbb{Z}} \mathfrak{g}_{p}: \mathfrak{r}=\bigoplus_{p \in \mathbb{Z}} \mathfrak{r}_{p}, \mathfrak{r}_{p}=\mathfrak{r} \cap \mathfrak{g}_{p}$. Since $\mathfrak{m}=\mathfrak{l}_{-}$, we see that $\mathfrak{r}_{-}=\{0\}$. By transitivity of $\mathfrak{g}=\bigoplus_{p \in \mathbb{Z}} \mathfrak{g}_{p}$, we get $\mathfrak{r}=\{0\}$, so $\mathfrak{g}$ is semisimple. Since dim $\mathfrak{g}_{p}=$ $\operatorname{dim} \mathfrak{g}_{-p}=\operatorname{dim} \mathfrak{l}_{-p}=\operatorname{dim} \mathfrak{l}_{p}$ for $p>0$, we get $\mathfrak{g}_{p}=\mathfrak{l}_{p}$ for $p \neq 0$. Since $\mathfrak{l}_{0}=\left[\mathfrak{l}_{-1}, \mathfrak{l}_{1}\right]=\left[\mathfrak{g}_{-1}, \mathfrak{g}_{1}\right]=\mathfrak{g}_{0}$, we obtain $\mathfrak{g}=\mathfrak{l}$.

The following lemma is essentially due to the proof of [2, Lemma 4.1].

Lemma 3.4. Let $(\mathfrak{m},[g])$ be a conformal pseudo-subriemannian $F G L A$ of type $(r, s)$, and let $\mathfrak{g}=$ $\bigoplus_{p \in \mathbb{Z}} \mathfrak{g}_{p}$ be the prolongation of $(\mathfrak{m},[g])$. If $\mathfrak{a}$ is a maximal $\mathbb{R}$-diagonalizable commutative subalgebra of $\mathfrak{g}$ contained in $\mathfrak{g}_{0}$, then $\operatorname{dim} \mathfrak{a} \leqq \min \{r, s\}+1$. In particular, if $\mathfrak{g}$ is semisimple, then we have $\operatorname{rank}_{\mathbb{R}} \mathfrak{g} \leqq \min \{r, s\}+1$.

Proof. Clearly $\mathfrak{a}$ contains the characteristic element $E$ of $\mathfrak{g}=\bigoplus_{p \in \mathbb{Z}} \mathfrak{g}_{p}$. By lemma 3.1, $\mathfrak{a}$ can be decomposed into the direct sum $\mathfrak{a}^{\prime} \oplus \mathbb{R} E$, where $\mathfrak{a}^{\prime}$ is a subalgebra of $\mathfrak{a}$ such that ad $\left(\mathfrak{a}^{\prime}\right) \mid \mathfrak{g}_{-1} \subset$ $\mathfrak{s o}\left(\mathfrak{g}_{-1}, g\right)$. Then $\mathfrak{a}^{\prime}$ is $\mathbb{R}$-diagonalizable in $\mathfrak{g}_{-1}$. Let $\lambda, \mu$ be weights of the $\mathfrak{a}^{\prime}$-module $\mathfrak{g}_{-1}$ and let $V^{\lambda}, V^{\mu}$ be the corresponding weight spaces. For $x \in V^{\lambda}, y \in V^{\mu}$ and $t \in \mathfrak{a}^{\prime}$, we get

$$
0=g([t, x], y)+g(x,[t, y])=(\lambda+\mu)(t) g(x, y) .
$$

Hence if $\lambda+\mu \neq 0$, then $g\left(V^{\lambda}, V^{\mu}\right)=0$. Let $\hat{\mathfrak{a}}$ be the subspace of $\mathfrak{a}^{*}$ spanned by the weights of the $\mathfrak{a}^{\prime}$-module $\mathfrak{g}_{-1}$. Since the $\mathfrak{a}^{\prime}$-module $\mathfrak{g}_{-1}$ is faithful, the annihilator space $\left\{h \in \mathfrak{a}^{\prime}: \lambda(h)=\right.$ 0 for all $\lambda \in \hat{\mathfrak{a}}\}$ vanishes, so $\operatorname{dim} \hat{\mathfrak{a}}=\operatorname{dim} \mathfrak{a}^{\prime}$. Thus the weights of the module span $\mathfrak{a}^{\prime *}$. There exists a basis $\left(\lambda_{1}, \ldots, \lambda_{l}\right)$ of $\mathfrak{a}^{\prime *}$ such that each $\lambda_{i}$ is a weight of the $\mathfrak{a}^{\prime}$-module $\mathfrak{g}_{-1}$. Then $U=\bigoplus_{i=1}^{l} V^{\lambda_{i}}$ 
is a totally isotropic subspace of $\left(\mathfrak{g}_{-1}, g\right)$, so $\operatorname{dim} \mathfrak{a}-1=\operatorname{dim} U \leqq \min \{r, s\}$. If $\mathfrak{g}$ is semisimple, then $\operatorname{rank}_{\mathbb{R}} \mathfrak{g}$ equals to $\operatorname{dim} \mathfrak{a}$, so we obtain $\operatorname{rank}_{\mathbb{R}} \mathfrak{g} \leqq \min \{r, s\}+1$.

\section{EXAmples of CONFORMAL PSEUdo-SUbriemannian FGLAs of SEMisimple type}

\subsection{Conformal pseudo-subriemannian FGLAs of classical type.}

Example 4.1 (cf. [12, §9] and [6, Example 3.1.2, p.241]). Let $\mathbb{K}$ be $\mathbb{C}, \mathbb{H}, \mathbb{C}^{\prime}$ or $\mathbb{H}^{\prime}$. Here we consider $\mathbb{K}$ as an $\mathbb{R}$-algebra. We put $\mathfrak{l}=\mathfrak{s l}(n, \mathbb{K})(n \geqq 3)$; then $\mathfrak{l}$ is a real simple Lie algebra. Let $K_{m}$ be the $m \times m$ matrix whose $(i, j)$-component is $\bar{\delta}_{i, m+1-j}$. We define an $n \times n$ symmetric real matrix $S_{p, q}$ as follows:

$$
S_{p, q}=\left[\begin{array}{ccc}
0 & 0 & K_{p} \\
0 & 1_{q} & 0 \\
K_{p} & 0 & 0
\end{array}\right] \quad(p \geqq 1, q \geqq 0,2 p+q=n \geqq 3),
$$

where $1_{q}$ denotes the $q \times q$ identity matrix. Here the center column and the center row of $S_{p, q}$ should be deleted when $q=0$. Then $S_{p, q}$ is a symmetric real matrix with signature $(p+q, p)$ such that $S_{p, q}^{2}=S_{p, q}$. We put $\mathfrak{g}=\left\{X \in \mathfrak{l}: X^{*} S_{p, q}+S_{p, q} X=O\right\}$; then

$$
\mathfrak{g}=\left\{X=\left[\begin{array}{ccc}
X_{11} & X_{12} & X_{13} \\
X_{21} & X_{22} & -S_{p-1, q} X_{12}^{*} \\
X_{31} & -X_{21}^{*} S_{p-1, q} & -X_{11}
\end{array}\right] \in \mathfrak{l}: \begin{array}{l}
X_{11} \in \mathbb{K}, X_{12} \in M\left(1, n^{\prime}, \mathbb{K}\right), \\
X_{31}, X_{13} \in \operatorname{Im} \mathbb{K}, X_{22} \in \mathfrak{g l}\left(n^{\prime}, \mathbb{K}\right), \\
X_{22}+S_{p-1, q} X_{22}^{*} S_{p-1, q}=O
\end{array}\right\},
$$

where $n^{\prime}=n-2$ and we set $S_{0, m}=1_{m}$. Here $M(p, q, \mathbb{K})$ denotes the set of $\mathbb{K}$-valued $p \times q$-matrices. We define subspaces $\mathfrak{g}_{p}$ of $\mathfrak{g}$ as follows:

$$
\begin{aligned}
& \mathfrak{g}_{-2}=\left\{\left[\begin{array}{ccc}
0 & 0 & 0 \\
0 & 0 & 0 \\
X_{31} & 0 & 0
\end{array}\right] \in \mathfrak{g}: X_{31} \in \operatorname{Im} \mathbb{K}\right\} \\
& \mathfrak{g}_{-1}=\left\{\left[\begin{array}{ccc}
0 & 0 & 0 \\
X_{21} & 0 & 0 \\
0 & -X_{21}^{*} S_{p-1, q} & 0
\end{array}\right] \in \mathfrak{g}: X_{21} \in M\left(n^{\prime}, 1, \mathbb{K}\right)\right\}, \\
& \mathfrak{g}_{0}=\left\{\left[\begin{array}{ccc}
X_{11} & 0 & 0 \\
0 & X_{22} & 0 \\
0 & 0 & -\overline{X_{11}}
\end{array}\right] \in \mathfrak{g}: \begin{array}{l}
X_{11} \in \mathbb{K}, X_{22} \in \mathfrak{g l}\left(n^{\prime}, \mathbb{K}\right), \\
X_{22}+S_{p-1, q} X_{22}^{*} S_{p-1, q}=O
\end{array}\right\}, \\
& \mathfrak{g}_{p}=\left\{X \in \mathfrak{g}:{ }^{t} X \in \mathfrak{g}_{-p}\right\} \quad(p=1,2), \quad \mathfrak{g}_{p}=\{0\} \quad(|p|>2) .
\end{aligned}
$$

Then $\mathfrak{g}=\bigoplus_{p \in \mathbb{Z}} \mathfrak{g}_{p}$ becomes a GLA whose negative part $\mathfrak{m}$ is an FGLA of the second kind. We define a symmetric bilinear form $g$ on $\mathfrak{g}_{-1}$ as follows:

$$
g(X, Y)=\operatorname{Re}\left(X_{21}^{*} S_{p-1, q} Y_{21}\right), \quad X, Y \in \mathfrak{g}_{-1} .
$$

Then $g$ is nondegenerate and for $A \in \mathfrak{g}_{0}$ we obtain $\left(\operatorname{ad}(A) \mid \mathfrak{g}_{-1}\right) \cdot g=-2\left(\operatorname{Re} A_{11}\right) g$. Hence $\operatorname{ad}\left(\mathfrak{g}_{0}\right) \mid \mathfrak{g}_{-1} \subset \mathfrak{c o}\left(\mathfrak{g}_{-1}, g\right)$. The conformal pseudo-subriemannian FGLA $(\mathfrak{m},[g])$ is said to be of type $(\mathrm{H} \mathbb{K})_{p, q}$.

In case $\mathbb{K}=\mathbb{C}$ or $\mathbb{C}^{\prime}$ we know that $\mathfrak{g}$ is denoted by $\mathfrak{s u}(p+q, p, \mathbb{K})(n=2 p+q)$. Note that $\mathfrak{s u}\left(p+q, p, \mathbb{C}^{\prime}\right)$ is isomorphic to $\mathfrak{s l}(2 p+q, \mathbb{R})$ for any $p, q$. If $\mathbb{K}=\mathbb{C}$ (resp. $\left.\mathbb{K}=\mathbb{C}^{\prime}\right)$, then $\mathfrak{g}=\bigoplus_{p \in \mathbb{Z}} \mathfrak{g}_{p}$ is a real SGLA of type $\left((\mathrm{AIIIa})_{l, p},\left\{\alpha_{1}, \alpha_{l}\right\}\right)(l=n-1=2 p+q-1, p \geqq 2, q \geqq 1),\left((\mathrm{AIIIb})_{l},\left\{\alpha_{1}, \alpha_{l}\right\}\right)$ $(l=n-1=2 p-1, p \geqq 2, q=0)$ or $\left((\mathrm{AIV})_{l},\left\{\alpha_{1}, \alpha_{l}\right\}\right)(l=n-1=q+1, p=1, q \geqq 1)$ (resp. $\left.\left((\mathrm{AI})_{l},\left\{\alpha_{1}, \alpha_{l}\right\}\right)(l=n-1=2 p+q-1 \geqq 2)\right)$, and $g$ has the signature $(2 p+2 q-2,2 p-2)=$ 
$(2 l-2 p, 2 p-2)(\operatorname{resp} .(2 p+q-2,2 p+q-2)=(l-1, l-1))$. Here $(\text { AIIIa })_{l, p},(\text { AIIIb })_{l},(\text { AIV })_{l}$ and $(\mathrm{AI})_{l}$ are the following Satake diagrams.

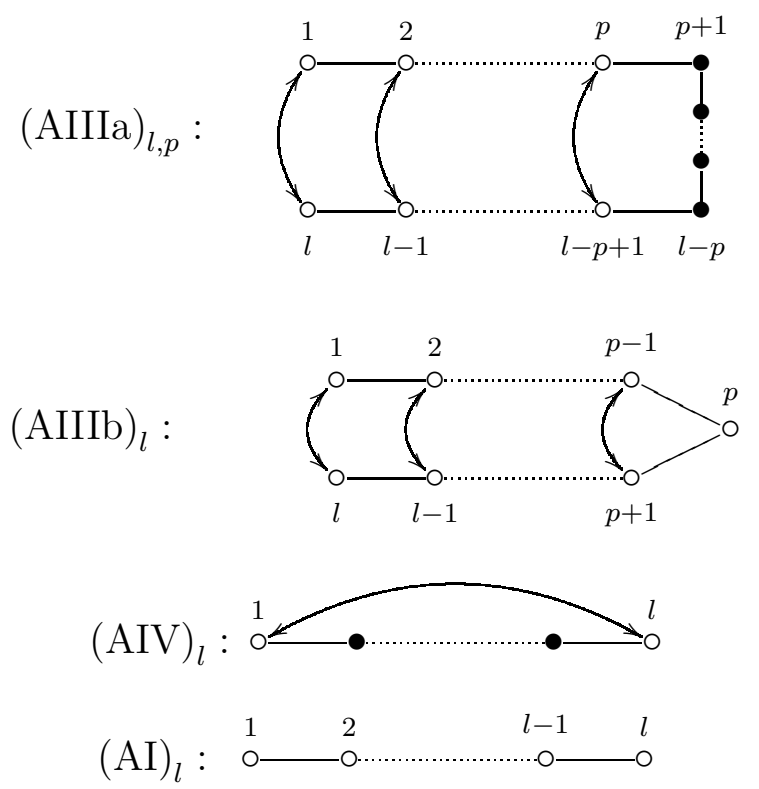

In case $\mathbb{K}=\mathbb{H}$ or $\mathbb{H}^{\prime}$ we know that $\mathfrak{g}$ is denoted by $\mathfrak{s p}(p+q, p, \mathbb{K})$. Note that $\mathfrak{s p}\left(p+q, p, \mathbb{H}^{\prime}\right)$ is isomorphic to $\mathfrak{s p}(2 p+q, \mathbb{R})$ for any $p, q$ and that $\mathfrak{s p}(n, \mathbb{R})$ is denoted by $\mathfrak{s p}(2 n, \mathbb{R})$ or $\mathfrak{s p} \mathfrak{p}_{2 n}(\mathbb{R})$ in [6], [9] and [11]. If $\mathbb{K}=\mathbb{H}$ (resp. $\left.\mathbb{K}=\mathbb{H}^{\prime}\right)$, then $\mathfrak{g}=\bigoplus_{p \in \mathbb{Z}} \mathfrak{g}_{p}$ is a real SGLA of type ((CIIa) $\left.)_{l, p},\left\{\alpha_{2}\right\}\right)$ $(l=n=2 p+q \geqq 3, p, q \geqq 1)$, or $\left((\mathrm{CIIb})_{l},\left\{\alpha_{2}\right\}\right)(n=l=2 p \geqq 3, q=0)\left(\operatorname{resp} . \quad\left((\mathrm{CI})_{l},\left\{\alpha_{2}\right\}\right)\right.$ $(l=n=2 p+q \geqq 3))$ and $g$ has the signature $(4 p+4 q-4,4 p-4)=(4 l-4 p-4,4 p-4)$ (resp. $(4 p+2 q-4,4 p+2 q-4)=(2 l-4,2 l-4))$. Here $(\mathrm{CIIa})_{l, p},(\mathrm{CIIb})_{l}$ and $(\mathrm{CI})_{l}$ are the following Satake diagrams.

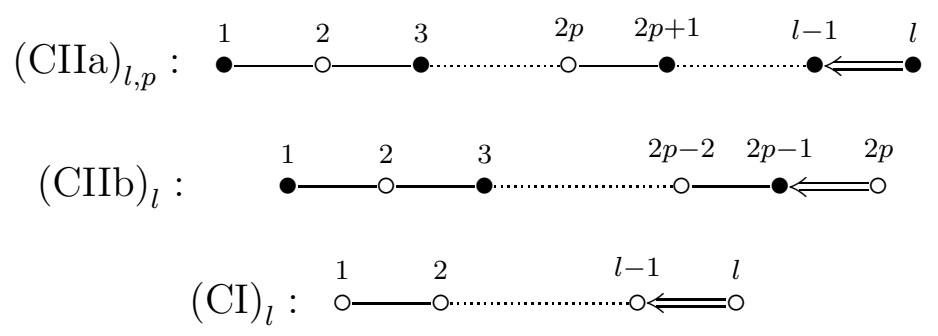

By Lemma 3.3, the prolongation of a conformal pseudo-subriemannian FGLA of type $(\mathrm{HK})_{p, q}$ coincides with $\mathfrak{g}=\bigoplus_{p \in \mathbb{Z}} \mathfrak{g}_{p}$.

Example 4.2 (cf.[15, $\S 4.4(3)])$. We put $\mathfrak{g}=\left\{X \in \mathfrak{g l}(2 l+1, \mathbb{R}):{ }^{t} X S+S X=0\right\}(l \geqq 2)$, where $S=S_{l, 1}$. More explicitly

$$
\mathfrak{g}=\left\{X=\left[\begin{array}{ccc}
A & a & B \\
\xi & 0 & -a^{\prime} \\
C & -\xi^{\prime} & -A^{\prime}
\end{array}\right] \in \mathfrak{g l}(2 l+1, \mathbb{R}): \begin{array}{l}
A, B, C \in \mathfrak{g l}(l, \mathbb{R}), \\
\\
a \in M(l, 1, \mathbb{R}), \xi \in M(1, l, \mathbb{R})
\end{array}\right\} .
$$

Here for an $r \times s$-matrix $X$ we put $X^{\prime}=K_{s}{ }^{t} X K_{r}$. The Lie algebra $\mathfrak{g}$ is a real simple Lie algebra $\mathfrak{s o}(l+1, l, \mathbb{R})$ of type $(\mathrm{BI})_{l, l}$. Here $(\mathrm{BI})_{l, l}$ is the following Satake diagram:

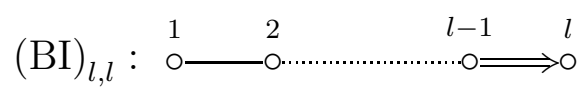


We define subspaces $\mathfrak{g}_{p}$ of $\mathfrak{g}$ as follows:

$$
\begin{aligned}
& \mathfrak{g}_{-3}=\left\{\left[\begin{array}{ccccc}
0 & 0 & 0 & 0 & 0 \\
0 & 0 & 0 & 0 & 0 \\
0 & 0 & 0 & 0 & 0 \\
X_{41} & 0 & 0 & 0 & 0 \\
0 & -X_{41}^{\prime} & 0 & 0 & 0
\end{array}\right] \in \mathfrak{g}: X_{41} \in M(l-1,1, \mathbb{R})\right\} \\
& \mathfrak{g}_{-2}=\left\{\left[\begin{array}{ccccc}
0 & 0 & 0 & 0 & 0 \\
0 & 0 & 0 & 0 & 0 \\
X_{31} & 0 & 0 & 0 & 0 \\
0 & X_{42} & 0 & 0 & 0 \\
0 & 0 & -X_{31} & 0 & 0
\end{array}\right] \in \mathfrak{g}: \begin{array}{l}
X_{31} \in \mathbb{R}, X_{42} \in \mathfrak{g l}(l-1, \mathbb{R}), \\
X_{42}^{\prime}=-X_{42}
\end{array}\right\} \\
& \mathfrak{g}_{-1}=\left\{\left[\begin{array}{ccccc}
0 & 0 & 0 & 0 & 0 \\
X_{21} & 0 & 0 & 0 & 0 \\
0 & X_{32} & 0 & 0 & 0 \\
0 & 0 & -X_{32}^{\prime} & 0 & 0 \\
0 & 0 & 0 & -X_{21}^{\prime} & 0
\end{array}\right] \in \mathfrak{g}: \begin{array}{c}
X_{21} \in M(l-1,1, \mathbb{R}), \\
X_{32} \in M(1, l-1, \mathbb{R})
\end{array}\right\} \\
& \mathfrak{g}_{0}=\left\{\left[\begin{array}{ccccc}
X_{11} & 0 & 0 & 0 & 0 \\
0 & X_{22} & 0 & 0 & 0 \\
0 & 0 & 0 & 0 & 0 \\
0 & 0 & 0 & -X_{22}^{\prime} & 0 \\
0 & 0 & 0 & 0 & -X_{11}
\end{array}\right] \in \mathfrak{g}: X_{11} \in \mathbb{R}, X_{22} \in \mathfrak{g l}(l-1, \mathbb{R})\right\}, \\
& \mathfrak{g}_{p}=\left\{X \in \mathfrak{g}:{ }^{t} X \in \mathfrak{g}_{-p}\right\} \quad(p=1,2,3), \quad \mathfrak{g}_{p}=\{0\} \quad(|p|>3) .
\end{aligned}
$$

Then $\mathfrak{g}=\bigoplus_{p \in \mathbb{Z}} \mathfrak{g}_{p}$ becomes a real SGLA of type $\left((\mathrm{BI})_{l, l},\left\{\alpha_{1}, \alpha_{l}\right\}\right)$ whose negative part $\mathfrak{m}$ is an FGLA of the third kind. We define a symmetric bilinear form $g$ on $\mathfrak{g}_{-1}$ by

$$
g(X, Y)=-\frac{1}{2}\left(X_{32} Y_{21}+Y_{32} X_{21}\right) \quad\left(X, Y \in \mathfrak{g}_{-1}\right)
$$

Then $g$ is nondegenerate, and for $A \in \mathfrak{g}_{0}$ we see that $\left(\operatorname{ad}(A) \mid \mathfrak{g}_{-1}\right) \cdot g=-A_{11} g$. Hence $\operatorname{ad}\left(\mathfrak{g}_{0}\right) \mid \mathfrak{g}_{-1} \subset$ $\mathfrak{c o}\left(\mathfrak{g}_{-1}, g\right)$. By Lemma $3.3(\mathfrak{m},[g])$ is a conformal neutral-subriemannian FGLA such that $\mathfrak{g}=\bigoplus_{p \in \mathbb{Z}} \mathfrak{g}_{p}$ is the prolongation of $(\mathfrak{m},[g])$. The conformal pseudo-subriemannian FGLA $(\mathfrak{m},[g])$ is said to be of type $(\mathrm{BI})_{l}$.

\subsection{Conformal pseudo-subriemannian FGLAs of exceptional type.}

Example $4.3([8, \S 3])$. Let $\mathbb{K}=\mathbb{O}$ or $\mathbb{O}^{\prime}$. Here we consider $\mathbb{K}$ as an $\mathbb{R}$-algebra. We define a nondegenerate symmetric bilinear form $g$ on $\mathbb{K}$ by $g(x, y)=\frac{1}{2}(\bar{x} y+\bar{y} x)$. We set

$$
\mathfrak{g}_{-1}=\mathfrak{g}_{1}=\mathbb{K}, \quad \mathfrak{g}_{-2}=\mathfrak{g}_{2}=\operatorname{Im} \mathbb{K}, \quad \mathfrak{g}_{0}=\mathfrak{g}_{0}^{\prime} \oplus \mathbb{R}, \quad \mathfrak{g}_{p}=\{0\} \quad \text { for }|p|>2,
$$

where $\mathfrak{g}_{0}^{\prime}=\{A \in \mathfrak{s o}(\mathbb{K}, g): A(1)=0\}$. Note that $\mathfrak{g}_{0}^{\prime}$ is isomorphic to $\mathfrak{s o}(\operatorname{Im} \mathbb{K}, g)$. We further put $\mathfrak{g}=\bigoplus_{p \in \mathbb{Z}} \mathfrak{g}_{p}$ and $\mathfrak{m}=\mathfrak{g}_{-2} \oplus \mathfrak{g}_{-1}$. We define a bracket operation $[\cdot, \cdot]$ on $\mathfrak{g}$ as in $[\mathbf{8}, \S 3.2$, p.444 and §3.4, pp.447-448]. By using [8, Lemma 3.1] we can prove that $\mathfrak{g}=\bigoplus_{p \in \mathbb{Z}} \mathfrak{g}_{p}$ becomes a GLA whose negative part $\mathfrak{m}$ is an FGLA of the second kind. For $A \oplus r \in \mathfrak{g}_{0}$ and $X, Y \in \mathfrak{g}_{-1}$ we see that $\left(\operatorname{ad}(A \oplus r) \mid \mathfrak{g}_{-1}\right) \cdot g=-2 r g$, and hence $\operatorname{ad}\left(\mathfrak{g}_{0}\right) \mid \mathfrak{g}_{-1} \subset \mathfrak{c o}\left(\mathfrak{g}_{-1}, g\right)$. The conformal pseudosubriemannian FGLA $(\mathfrak{m},[g])$ is said to be of type $(\mathrm{HK})$. 
By $\left[\mathbf{8}\right.$, Theorem 3.5], in case $\mathbb{K}=\mathbb{O}$ (resp. $\mathbb{K}=\mathbb{O}^{\prime}$ ) the GLA $\mathfrak{g}=\bigoplus_{p \in \mathbb{Z}} \mathfrak{g}_{p}$ is a real SGLA of type (FII, $\left.\left\{\alpha_{4}\right\}\right)$ (resp. $\left.\left(\mathrm{FI},\left\{\alpha_{4}\right\}\right)\right)$. Here $\mathrm{FII}\left(=\mathrm{F}_{4(-20)}\right)$ and $\mathrm{FI}\left(=\mathrm{F}_{4(4)}\right)$ are the following Satake diagrams respectively.

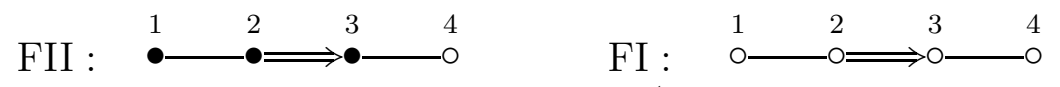

Clearly $(\mathfrak{m},[g])$ is a conformal subriemannian FGLA (resp. a conformal neutral-subriemannian FGLA) when $\mathbb{K}=\mathbb{O}$ (resp. $\mathbb{K}=\mathbb{O}^{\prime}$ ). By Lemma 3.3, the prolongation of a conformal pseudosubriemannian FGLA of type $(\mathrm{HK})$ coincides with $\mathfrak{g}=\bigoplus_{p \in \mathbb{Z}} \mathfrak{g}_{p}$.

Example 4.4. Let $V$ be a real vector space $\mathbb{R}^{2}$, and we set $\mathfrak{s}=\mathfrak{s l}(V)$. We define real vector spaces $\mathfrak{l}_{p}(p \in \mathbb{Z})$ as follows:

$$
\mathfrak{l}_{-2}=\mathfrak{l}_{2}=\mathbb{R}, \quad \mathfrak{l}_{-1}=\mathfrak{l}_{1}=S^{3}(V), \quad \mathfrak{l}_{0}=\mathfrak{s} \oplus \mathbb{R}, \quad \mathfrak{l}_{p}=\{0\} \quad(p>|2|) .
$$

We define a bracket operation $[\cdot, \cdot]$ on $\mathfrak{l}=\bigoplus_{p \in \mathbb{Z}} \mathfrak{l}_{p}$ as in $[\mathbf{8}, \S 3.1, \mathrm{p} .450]$. Then $\mathfrak{l}=\bigoplus_{p \in \mathbb{Z}} \mathfrak{l}_{p}$ becomes a real SGLA of type $\left(\mathrm{G}_{2(2)},\left\{\alpha_{2}\right\}\right)([8$, Theorem 4.3]) and the negative part $\mathfrak{m}$ is an FGLA of the second kind. Here $\mathrm{G}_{2(2)}$ is the following Satake diagram:

$$
\mathrm{G}_{2(2)}: \quad \stackrel{1}{\circ}{ }^{2}
$$

We set $V_{-1}=\mathbb{R} e_{1}$ and $V_{-2}=\mathbb{R} e_{2}$, where $\left(e_{1}, e_{2}\right)$ is the canonical basis of $V$. We put $\mathfrak{s}_{p}=\{X \in$ $\mathfrak{s}: X\left(V_{k}\right) \subset V_{k+p}$ for all $\left.k\right\}$; then $\mathfrak{s}=\bigoplus_{p \in \mathbb{Z}} \mathfrak{s}_{p}$ is a real SGLA of the first kind.

We define subspaces $W_{k}$ (resp. $\left.W_{-k}\right)(k=1, \ldots, 4)$ of $\mathfrak{l}_{1}\left(\right.$ resp. $\left.\mathfrak{l}_{-1}\right)$ as follows:

$$
W_{ \pm 1}=S^{3}\left(V_{-1}\right), \quad W_{ \pm 2}=S^{2}\left(V_{-1}\right) \otimes V_{-2}, \quad W_{ \pm 3}=V_{-1} \otimes S^{2}\left(V_{-2}\right), \quad W_{ \pm 4}=S^{3}\left(V_{-2}\right)
$$

then $\mathfrak{l}_{ \pm 1}=\bigoplus_{k=1}^{4} W_{ \pm k}$. We define subspaces $\mathfrak{g}_{p}$ of $\mathfrak{l}$ as follows:

$$
\begin{aligned}
& \mathfrak{g}_{ \pm 5}=\mathfrak{l}_{ \pm 2}, \quad \mathfrak{g}_{k}=W_{k} \quad(k= \pm 2, \pm 3, \pm 4), \quad \mathfrak{g}_{ \pm 1}=\mathfrak{s}_{ \pm 1} \oplus W_{ \pm 1} \\
& \mathfrak{g}_{0}=\mathfrak{s}_{0} \oplus \mathbb{R}, \quad \mathfrak{g}_{p}=\{0\} \quad(p>|5|) .
\end{aligned}
$$

Then $\mathfrak{l}=\mathfrak{g}=\bigoplus_{p \in \mathbb{Z}} \mathfrak{g}_{p}$ becomes a real SGLA of type $\left(\mathrm{G}_{2(2)},\left\{\alpha_{1}, \alpha_{2}\right\}\right)$ such that the negative part $\mathfrak{m}$ is an FGLA of the 5-th kind. Let $(\cdot \mid \cdot)$ be an inner product on $S^{3}(V)$ induced by the canonical inner product on $V$. We define a symmetric bilinear form $g$ on $\mathfrak{g}_{-1}$ as follows:

$$
g\left(\mathfrak{s}_{-1}, \mathfrak{s}_{-1}\right)=g\left(W_{-1}, W_{-1}\right)=0, \quad g(X, u)=g(u, X)=\left(X u \mid e_{1}^{2} e_{2}\right) \quad\left(X \in \mathfrak{s}_{-1}, u \in W_{-1}\right) .
$$

Then $g$ is nondegenerate, and for $A=\lambda\left(E_{11}-E_{22}\right) \oplus r \in \mathfrak{g}_{0}, X \in \mathfrak{s}_{-1}$ and $u \in W_{-1}(\lambda, r \in \mathbb{R})$, we see that $\left(\operatorname{ad}(A) \mid \mathfrak{g}_{-1}\right) \cdot g=(\lambda-r) g$, where $E_{i j}$ is an element of $\mathfrak{s}$ such that $E_{i j} e_{k}=\delta_{j k} e_{i}$. Thus $\operatorname{ad}\left(\mathfrak{g}_{0}\right) \mid \mathfrak{g}_{-1} \subset \mathfrak{c o}\left(\mathfrak{g}_{-1}, g\right)$. Hence by Lemma $3.3(\mathfrak{m},[g])$ is a conformal neutral-subriemannian FGLA such that $\mathfrak{g}=\bigoplus_{p \in \mathbb{Z}} \mathfrak{g}_{p}$ is the prolongation of $(\mathfrak{m},[g])$. The conformal pseudo-subriemannian FGLA $(\mathfrak{m},[g])$ is said to be of type $(\mathrm{G})$.

\section{Classification of Conformal pseudo-subriemannian FGLAs of Semisimple type}

In this section we prove that a conformal pseudo-subriemannian FGLA of semisimple type is isomorphic to one of conformal pseudo-subriemannian FGLAs given in the previous section.

Proposition 5.1. Let $(\mathfrak{m},[g])$ be a conformal pseudo-subriemannian FGLA of semisimple type, and let $\mathfrak{g}=\bigoplus_{p \in \mathbb{Z}} \mathfrak{g}_{p}$ be the prolongation of $(\mathfrak{m},[g])$. 
(1) If the $\mathfrak{g}_{0}$-module $\mathfrak{g}_{-1}$ is irreducible and the $\mathfrak{g}_{0}(\mathbb{C})$-module $\mathfrak{g}_{-1}(\mathbb{C})$ is reducible, there exist $\mathfrak{g}_{0}(\mathbb{C})$-submodules $\mathfrak{g}_{-1}(\mathbb{C})^{(i)}(i=1,2)$ such that $(\mathrm{i}) \mathfrak{g}_{-1}(\mathbb{C})=\mathfrak{g}_{-1}(\mathbb{C})^{(1)} \oplus \mathfrak{g}_{-1}(\mathbb{C})^{(2)} ;$ (ii) $\mathfrak{g}_{-1}(\mathbb{C})^{(i)}(i=1,2)$ are totally isotropic subspaces of $\left(\mathfrak{g}_{-1}(\mathbb{C}), g\right)$; (iii) $\mathfrak{g}_{-1}(\mathbb{C})^{(1)}$ is contragredient to $\mathfrak{g}_{-1}(\mathbb{C})^{(2)}$ as a $\hat{\mathfrak{g}}_{0}(\mathbb{C})$-module, where $\hat{\mathfrak{g}}_{0}=\left(\rho_{-1}\right)^{-1}\left(\mathfrak{s o}\left(\mathfrak{g}_{-1}, g\right)\right)$.

(2) If the $\mathfrak{g}_{0}$-module $\mathfrak{g}_{-1}$ is reducible, then there exist $\mathfrak{g}_{0}$-submodules $\mathfrak{g}_{-1}^{(i)}(i=1,2)$ such that: (i) $\mathfrak{g}_{-1}=\mathfrak{g}_{-1}^{(1)} \oplus \mathfrak{g}_{-1}^{(2)}$; (ii) $\mathfrak{g}_{-1}^{(i)}(i=1,2)$ are totally isotropic subspaces of $\left(\mathfrak{g}_{-1}, g\right)$; (iii) $\mathfrak{g}_{-1}^{(1)}$ is contragredient to $\mathfrak{g}_{-1}^{(2)}$ as a $\hat{\mathfrak{g}}_{0}$-module; (iv) the $\mathfrak{g}_{0}(\mathbb{C})$-modules $\mathfrak{g}_{-1}^{(i)}(\mathbb{C})$ are irreducible.

(3) $\mathfrak{g}=\bigoplus_{p \in \mathbb{Z}} \mathfrak{g}_{p}$ is an SGLA of class $(\mathrm{B})$.

Proof. (1) and $(2)$. We decompose $\mathfrak{g}_{-1}\left(\right.$ resp. $\left.\mathfrak{g}_{-1}(\mathbb{C})\right)$ into irreducible $\mathfrak{g}_{0}$-modules (resp. $\mathfrak{g}_{0}(\mathbb{C})$ modules) as follows:

$$
\mathfrak{g}_{-1}=\bigoplus_{i=1}^{k} \mathfrak{g}_{-1}^{(i)}, \quad \mathfrak{g}_{-1}(\mathbb{C})=\bigoplus_{i=1}^{k^{\prime}} \mathfrak{g}_{-1}(\mathbb{C})^{(i)}
$$

Let $E_{i}$ be an element of $\mathfrak{g}_{0}$ such that $\left[E_{i}, X_{j}\right]=-\delta_{i j} X_{j}$ for all $X_{j} \in \mathfrak{g}_{-1}^{(j)}$. We first assume that $\mathfrak{g}_{-1}^{(1)}$ is a nondegenerate subspace of $\left(\mathfrak{g}_{-1}, g\right)$. There exist elements $X_{1}, Y_{1}$ of $\mathfrak{g}_{-1}^{(1)}$ such that $g\left(X_{1}, Y_{1}\right) \neq 0$. Since

$$
g\left(\left[E_{1}, X_{1}\right], Y_{1}\right)+g\left(X_{1},\left[E_{1}, Y_{1}\right]\right)=\eta_{E_{1}} g\left(X_{1}, Y_{1}\right)
$$

we see that $\eta_{E_{1}}=-2$. For $X_{i} \in \mathfrak{g}_{-1}^{(i)}(i \geqq 2)$,

$$
g\left(\left[E_{1}, X_{1}\right], X_{i}\right)+g\left(X_{1},\left[E_{1}, X_{i}\right]\right)=\eta_{E_{1}} g\left(X_{1}, X_{i}\right),
$$

so $g\left(X_{1}, X_{i}\right)=0$. Thus we get $g\left(\mathfrak{g}_{-1}^{(1)}, \mathfrak{g}_{-1}^{(i)}\right)=0(i \geqq 2)$. If there exists a $j \geqq 2$ such that $\mathfrak{g}_{-1}^{(j)}$ is a nondegenerate subspace of $\left(\mathfrak{g}_{-1}, g\right)$, then

$$
0=g\left(\left[E_{j}, X_{1}\right], Y_{1}\right)+g\left(X_{1},\left[E_{j}, Y_{1}\right]\right)=\eta_{E_{j}} g\left(X_{1}, Y_{1}\right)=-2 g\left(X_{1}, Y_{1}\right)
$$

which is a contradiction. Hence $\mathfrak{g}_{-1}^{(i)}(i \geqq 2)$ are totally isotropic subspaces of $\left(\mathfrak{g}_{-1}, g\right)$. Assume that $\mathfrak{g}_{-1}^{(2)} \neq\{0\}$. There exists $j \geqq 3$ such that the restriction of $g$ to the space $\mathfrak{g}_{-1}^{(1)} \times \mathfrak{g}_{-1}^{(j)}$ is nondegenerate. We set $E_{2}^{\prime}=E_{2}+E_{j}$. Let $X_{2}$ (resp. $\left.X_{j}\right)$ be an element of $\mathfrak{g}_{-1}^{(2)}\left(\right.$ resp. $\left.\mathfrak{g}_{-1}^{(j)}\right)$ such that $g\left(X_{2}, X_{j}\right) \neq 0$. Since

$$
g\left(\left[E_{2}^{\prime}, X_{2}\right], X_{j}\right)+g\left(X_{2},\left[E_{2}^{\prime}, X_{j}\right]\right)=\eta_{E_{2}^{\prime}} g\left(X_{2}, X_{j}\right),
$$

we see that $\eta_{E_{2}^{\prime}}=-2$. Also

$$
0=g\left(\left[E_{2}^{\prime}, X_{1}\right], Y_{1}\right)+g\left(X_{1},\left[E_{2}^{\prime}, Y_{1}\right]\right)=-2 g\left(X_{1}, Y_{1}\right)
$$

This is a contradiction. Therefore we obtain that $\mathfrak{g}_{-1}$ is an irreducible $\mathfrak{g}_{0}$-module. Next we assume that $\mathfrak{g}_{-1}^{(i)}$ is a totally isotropic subspace of $\left(\mathfrak{g}_{-1}, g\right)$. Here we may assume that the restriction of $g$ to $\mathfrak{g}_{-1}^{(1)} \times \mathfrak{g}_{-1}^{(2)}$ is nondegenerate. From the above result, $\mathfrak{g}_{-1}^{(2)}$ is a totally isotropic subspace of $\left(\mathfrak{g}_{-1}, g\right)$ and is contragredient to $\mathfrak{g}_{-1}^{(1)}$ as a $\hat{\mathfrak{g}}_{0}$-module. If the restriction of $g$ to $\mathfrak{g}_{-1}^{(1)} \times \mathfrak{g}_{-1}^{(3)}$ is nondegenerate, $\mathfrak{g}_{-1}^{(3)}$ is contragredient to $\mathfrak{g}_{-1}^{(1)}$ as a $\hat{\mathfrak{g}}_{0}$-module, so $\mathfrak{g}_{-1}^{(1)}$ is isomorphic to $\mathfrak{g}_{-1}^{(3)}$ as a $\mathfrak{g}_{0}$-module, which is a contradiction. Hence $g\left(\mathfrak{g}_{-1}^{(1)}, \mathfrak{g}_{-1}^{(3)}\right)=0$. Similarly we get $g\left(\mathfrak{g}_{-1}^{(2)}, \mathfrak{g}_{-1}^{(3)}\right)=0$. There exists $k \geqq 4$ such that the restriction of $g$ to $\mathfrak{g}_{-1}^{(3)} \times \mathfrak{g}_{-1}^{(k)}$ is nondegenerate. We set $E_{1}^{\prime}=E_{1}+E_{2}$. Let $X_{i}(i=1,2,3, k)$ be elements of $\mathfrak{g}_{-1}^{(i)}$ such that $g\left(X_{1}, X_{2}\right) \neq 0$ and $g\left(X_{3}, X_{k}\right) \neq 0$. Since

$$
0=g\left(\left[E_{1}^{\prime}, X_{1}\right], X_{2}\right)+g\left(X_{1},\left[E_{1}^{\prime}, X_{2}\right]\right)=\eta_{E_{1}^{\prime}} g\left(X_{1}, X_{2}\right),
$$

we get $\eta_{E_{1}^{\prime}}=-2$. On the other hand, we see that

$$
0=g\left(\left[E_{1}^{\prime}, X_{3}\right], X_{k}\right)+g\left(X_{3},\left[E_{1}^{\prime}, X_{k}\right]\right)=\eta_{E_{1}^{\prime}} g\left(X_{3}, X_{k}\right)
$$


which is a contradiction. Hence $\mathfrak{g}_{-1}=\mathfrak{g}_{-1}^{(1)} \oplus \mathfrak{g}_{-1}^{(2)}$. Similarly we can prove that if the $\mathfrak{g}_{0}(\mathbb{C})$-module $\mathfrak{g}_{-1}(\mathbb{C})$ is reducible, there exist $\mathfrak{g}_{0}(\mathbb{C})$-submodules $\mathfrak{g}_{-1}(\mathbb{C})^{(i)}(i=1,2)$ such that $(\mathrm{i}) \mathfrak{g}_{-1}(\mathbb{C})=$ $\mathfrak{g}_{-1}(\mathbb{C})^{(1)} \oplus \mathfrak{g}_{-1}(\mathbb{C})^{(2)} ;$ (ii) $\mathfrak{g}_{-1}(\mathbb{C})^{(i)}(i=1,2)$ are totally isotropic subspaces of $\left(\mathfrak{g}_{-1}(\mathbb{C}), g\right) ;($ iii $)$ $\mathfrak{g}_{-1}(\mathbb{C})^{(1)}$ is contragredient to $\mathfrak{g}_{-1}(\mathbb{C})^{(2)}$ as a $\hat{\mathfrak{g}}_{0}(\mathbb{C})$-module. The assertions $(1)$ and $(2)$ follow from these results.

(3) We assume that $\mathfrak{g}$ is not simple. There exist ideals $\mathfrak{a}^{(1)}$ and $\mathfrak{a}^{(2)}$ of $\mathfrak{g}$ such that $\mathfrak{a}^{(1)}$ is a simple ideal of $\mathfrak{g}$ and $\mathfrak{g}=\mathfrak{a}^{(1)} \oplus \mathfrak{a}^{(2)}$. Both ideals $\mathfrak{a}^{(i)}(i=1,2)$ are graded ideals of $\mathfrak{g}$; we write $\mathfrak{a}^{(i)}=\bigoplus_{p \in \mathbb{Z}} \mathfrak{a}_{p}^{(i)}$. By transitivity of $\mathfrak{g}=\bigoplus_{p \in \mathbb{Z}} \mathfrak{g}_{p}$, we see that $\mathfrak{a}_{-1}^{(i)} \neq\{0\}(i=1,2)$. From the results of $(1)$ and $(2) \mathfrak{a}_{-1}^{(2)}$ is contragredient to $\mathfrak{a}_{-1}^{(1)}$ as a $\hat{\mathfrak{g}}_{0}$-module, which is a contradiction. Hence $\mathfrak{g}$ is simple. Also from the results of (1) and (2) and from [9, p.157, Example 2], it follows that $\mathfrak{g}$ is of class (B).

We decompose the conformal pseudo-subriemannian FGLAs of semisimple type into the following three classes:

(SI) The $\mathfrak{g}_{0}(\mathbb{C})$-module $\mathfrak{g}_{-1}(\mathbb{C})$ is irreducible.

(SII) The $\mathfrak{g}_{0}$-module $\mathfrak{g}_{-1}$ is irreducible and the $\mathfrak{g}_{0}(\mathbb{C})$-module $\mathfrak{g}_{-1}(\mathbb{C})$ is reducible.

(SIII) The $\mathfrak{g}_{0}$-module $\mathfrak{g}_{-1}$ is reducible.

Theorem 5.1. Let $(\mathfrak{m},[g])$ be a conformal pseudo-subriemannian FGLA of semisimple type, and let $\mathfrak{g}=\bigoplus_{p \in \mathbb{Z}} \mathfrak{g}_{p}$ be the prolongation of $(\mathfrak{m},[g])$. Assume that $(\mathfrak{m},[g])$ is of type $(r, s)(r \geqq s)$.

(1) If $(\mathfrak{m},[g])$ is of class $(\mathrm{SI})$, then $\mathfrak{g}=\bigoplus_{p \in \mathbb{Z}} \mathfrak{g}_{p}$ is an $S G L A$ of type $\left((\mathrm{CI})_{l},\left\{\alpha_{2}\right\}\right)$, ((CIIa) $\left.)_{l, p},\left\{\alpha_{2}\right\}\right)$, $\left((\mathrm{CIIb})_{l},\left\{\alpha_{2}\right\}\right)(l \geqq 3, p \geqq 1)$, (FI, $\left.\left\{\alpha_{4}\right\}\right)$, or (FII, $\left.\left\{\alpha_{4}\right\}\right)$.

(2) If $(\mathfrak{m},[g])$ is of class (SII), then $\mathfrak{g}=\bigoplus_{p \in \mathbb{Z}} \mathfrak{g}_{p}$ is an $S G L A$ of type ((AIIIa) $\left.)_{l, p},\left\{\alpha_{1}, \alpha_{l}\right\}\right)$, $\left((\mathrm{AIIIb})_{l},\left\{\alpha_{1}, \alpha_{l}\right\}\right)$ or $\left((\mathrm{AIV})_{l},\left\{\alpha_{1}, \alpha_{l}\right\}\right)(l \geqq 2)$.

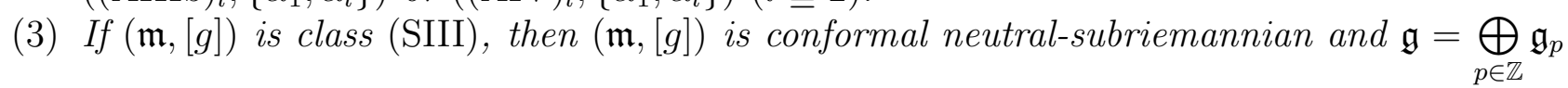
is an $S G L A$ of type $\left((\mathrm{AI})_{l},\left\{\alpha_{1}, \alpha_{l}\right\}\right),\left((\mathrm{BI})_{l, l},\left\{\alpha_{1}, \alpha_{l}\right\}\right)(l \geqq 2)$ or $\left(\mathrm{G}_{2(2)},\left\{\alpha_{1}, \alpha_{2}\right\}\right)$.

Proof. By Proposition 5.1 the complexification $\mathfrak{g}(\mathbb{C})=\bigoplus_{p \in \mathbb{Z}} \mathfrak{g}_{p}(\mathbb{C})$ is an SGLA. We first assume that $(\mathfrak{m},[g])$ is of class $(\mathrm{SI}) ;$ then $\mathfrak{g}(\mathbb{C})=\bigoplus_{p \in \mathbb{Z}} \mathfrak{g}_{p}(\mathbb{C})$ be of type $\left(X_{l},\left\{\alpha_{i}\right\}\right)$. Furthermore $\mathfrak{g}_{-1}(\mathbb{C})$ is an irreducible $\mathfrak{g}_{0}(\mathbb{C})$-module with highest weight $-\alpha_{i}$ and there exists a $\mathfrak{g}_{0}^{s s}(\mathbb{C})$-invariant symmetric bilinear form $g$ on $\mathfrak{g}_{-1}(\mathbb{C})$. By Proposition $2.2(1)$ we obtain that $\left(X_{l},\left\{\alpha_{i}\right\}\right)$ is one of $\left(C_{l},\left\{\alpha_{2}\right\}\right)$ $(l \geqq 3),\left(F_{4},\left\{\alpha_{4}\right\}\right)$. Next we assume that $(\mathfrak{m},[g])$ is of class (SII) or (SIII). By Proposition 5.1 $(2)$, the $\mathfrak{g}_{0}(\mathbb{C})$-module $\mathfrak{g}_{-1}(\mathbb{C})$ is decomposed as follows: $\mathfrak{g}_{-1}(\mathbb{C})=\mathfrak{g}_{-1}(\mathbb{C})^{(1)} \oplus \mathfrak{g}_{-1}(\mathbb{C})^{(2)}$, where $\mathfrak{g}_{-1}(\mathbb{C})^{(i)}(i=1,2)$ are irreducible $\mathfrak{g}_{0}(\mathbb{C})$-submodule of $\mathfrak{g}_{-1}(\mathbb{C})$ such that: (i) each $\mathfrak{g}_{-1}(\mathbb{C})^{(i)}$ is totally isotropic with respect to $g ;\left(\right.$ ii) $\mathfrak{g}_{-1}(\mathbb{C})^{(1)}$ is contragredient to $\mathfrak{g}_{-1}(\mathbb{C})^{(2)}$ as a $\hat{\mathfrak{g}}_{0}(\mathbb{C})$-module. By Proposition $2.2(2)$ and Remark 2.1, we obtain that $\mathfrak{g}(\mathbb{C})=\bigoplus_{p \in \mathbb{Z}} \mathfrak{g}_{p}(\mathbb{C})$ is of type $\left(A_{l},\left\{\alpha_{1}, \alpha_{l}\right\}\right)$, $\left(B_{l},\left\{\alpha_{1}, \alpha_{l}\right\}\right)$ or $\left(G_{2},\left\{\alpha_{1}, \alpha_{2}\right\}\right)$. Hence the assertions (1)-(3) follow from Proposition 2.4, and the tables of [11, pp.79-82] and [14, pp.30-32].

Proposition 5.2. Let $\left(\mathfrak{m},\left[g_{1}\right]\right)$ and $\left(\mathfrak{m},\left[g_{2}\right]\right)$ be two conformal pseudo-subriemannian FGLAs of semisimple type. If the prolongation of $\left(\mathfrak{m},\left[g_{1}\right]\right)$ coincides with that of $\left(\mathfrak{m},\left[g_{2}\right]\right)$, then $\left(\mathfrak{m},\left[g_{1}\right]\right)$ is equivalent to $\left(\mathfrak{m},\left[g_{2}\right]\right)$.

Proof. The mapping $\varphi=g_{1}^{\sharp} \circ g_{2}^{b}$ induces an isomorphism of $\mathfrak{g}_{-1}(\mathbb{C})$ onto itself as a $\hat{\mathfrak{g}}_{0}(\mathbb{C})$-module. If the $\mathfrak{g}_{0}(\mathbb{C})$-module $\mathfrak{g}_{-1}(\mathbb{C})$ is reducible, then $\mathfrak{g}_{-1}(\mathbb{C})$ is the direct sum of two irreducible $\mathfrak{g}_{0}(\mathbb{C})$ modules $\mathfrak{g}_{-1}(\mathbb{C})^{(i)}(i=1,2)$ and $\mathfrak{g}_{-1}(\mathbb{C})^{(1)}$ is not isomorphic to $\mathfrak{g}_{-1}(\mathbb{C})^{(2)}$ as a $\mathfrak{g}_{0}(\mathbb{C})$-module. In 
this case $\varphi\left(\mathfrak{g}_{-1}(\mathbb{C})^{(i)}\right)=\mathfrak{g}_{-1}(\mathbb{C})^{(i)}(i=1,2)$. By Schur's lemma, there exist two complex numbers $\lambda_{1}, \lambda_{2}$ such that $\varphi \mid \mathfrak{g}_{-1}(\mathbb{C})^{(i)}=\lambda_{i} i d(i=1,2)$. For $X \in \mathfrak{g}_{-1}(\mathbb{C})^{(1)}$ and $Y \in \mathfrak{g}_{-1}(\mathbb{C})^{(2)}$ we obtain $\lambda_{1} g_{1}(X, Y)=g_{1}(\varphi(X), Y)=g_{2}(X, Y)$ and $\lambda_{2} g_{1}(X, Y)=g_{1}(X, \varphi(Y))=g_{2}(X, Y)$. Since $\mathfrak{g}_{-1}(\mathbb{C})^{(i)}$ are totally isotropic with respect to $g_{1}$ and $g_{2}$, we get $\lambda_{1}=\lambda_{2}$. Hence $g_{2}=\lambda_{1} g_{1}$ and $\lambda_{i} \in \mathbb{R}$. Thus we see that $\left[g_{1}\right]=\left[g_{2}\right]$ or $\left[g_{1}\right]=\left[-g_{2}\right]$. Similarly we can prove that $\left[g_{1}\right]=\left[g_{2}\right]$ or $\left[g_{1}\right]=\left[-g_{2}\right]$ when the $\mathfrak{g}_{0}(\mathbb{C})$-module $\mathfrak{g}_{-1}(\mathbb{C})$ is irreducible.

From Theorem 5.1, Proposition 5.2 and the results of $\S 4$ we obtain the following theorem.

Theorem 5.2. Let $(\mathfrak{m},[g])$ be a conformal pseudo-subriemannian FGLA of semisimple type. Then $(\mathfrak{m},[g])$ is equivalent to one of conformal pseudo-subriemannian $F G L A$ s of types $(\mathrm{H} \mathbb{C})_{p, q},\left(\mathrm{H} \mathbb{C}^{\prime}\right)_{p, q}$, $(\mathrm{HH})_{p, q},\left(\mathrm{HH}^{\prime}\right)_{p, q},(\mathrm{HO}),\left(\mathrm{HO}{ }^{\prime}\right),(\mathrm{BI})_{l},(\mathrm{G})$. The prolongation $\mathfrak{g}=\bigoplus_{p \in \mathbb{Z}} \mathfrak{g}_{p}$ of $(\mathfrak{m},[g])$ and the signature $(r, s)$ of $g$ are given in the following table.

\begin{tabular}{|c|l|l|l|l|}
\hline$(\mathfrak{m},[g])$ & $(\mathrm{H} \mathbb{C})_{p, q}(p \geqq 2, q \geqq$ & $(\mathrm{H} \mathbb{C})_{p, 0}(p \geqq 2)$ & $(\mathrm{HC})_{1, q}(q \geqq 1)$ & $\begin{array}{l}\left(\mathrm{H} \mathbb{C}^{\prime}\right)_{p, q} \quad(p \geqq 1, \\
2 p+q \geqq 3)\end{array}$ \\
\hline $\mathfrak{g}=\bigoplus_{p \in \mathbb{Z}} \mathfrak{g}_{p}$ & $\begin{array}{l}\left.(\mathrm{AIIIa})_{l, p},\left\{\alpha_{1}, \alpha_{l}\right\}\right) \\
(l=2 p+q-1)\end{array}$ & $\begin{array}{l}\left.(\mathrm{AIIIb})_{l},\left\{\alpha_{1}, \alpha_{l}\right\}\right) \\
(l=2 p-1)\end{array}$ & $\begin{array}{l}\left((\mathrm{AIV})_{l},\left\{\alpha_{1}, \alpha_{l}\right\}\right) \\
(l=q+1)\end{array}$ & $\begin{array}{l}\left((\mathrm{AI})_{l},\left\{\alpha_{1}, \alpha_{l}\right\}\right) \\
(l=2 p+q-1)\end{array}$ \\
\hline$(r, s)$ & $(2 p+2 q-2,2 p-2)$ & $(2 p-2,2 p-2)$ & $(2 q, 0)$ & $\begin{array}{l}(2 p+q-2,2 p+ \\
q-2)\end{array}$ \\
\hline \hline$(\mathfrak{m},[g])$ & $(\mathrm{HHI})_{p, q}(p, q \geqq 1)$ & $(\mathrm{HHI})_{p, 0}(p \geqq 2)$ & $\begin{array}{l}\left(\mathrm{H} \mathbb{H}^{\prime}\right)_{p, q}(p \geqq 1, \\
2 p+q \geqq 3)\end{array}$ & $(\mathrm{HO})$ \\
\hline $\mathfrak{g}=\bigoplus_{p \in \mathbb{Z}} \mathfrak{g}_{p}$ & $\left((\mathrm{CIIa})_{l, p},\left\{\alpha_{2}\right\}\right)$ & $\left((\mathrm{CIIb})_{l},\left\{\alpha_{2}\right\}\right)$ & $\begin{array}{l}\left((\mathrm{CI})_{l},\left\{\alpha_{2}\right\}\right)(l= \\
2 p+q)\end{array}$ & $\left(\mathrm{FII},\left\{\alpha_{4}\right\}\right)$ \\
\hline$(r, s)$ & $(l=2 p+q)$ & $(l=2 p)$ & $(4 p+2 q-4,4 p+$ & $(8,0)$ \\
\hline \hline$(\mathfrak{m},[g])$ & $\left(\mathrm{H} \mathbb{O}^{\prime}\right)$ & $(4 p-4,4 p-4)$ & $2 q-4)$ & \\
\hline $\mathfrak{g}=\bigoplus_{p \in \mathbb{Z}} \mathfrak{g}_{p}$ & $\left(\mathrm{FI},\left\{\alpha_{4}\right\}\right)$ & $(\mathrm{BI})_{l}(l \geqq 2)$ & $(\mathrm{G})$ & $\left(\mathrm{G}_{2(2)},\left\{\alpha_{1}, \alpha_{2}\right\}\right)$ \\
\hline$(r, s)$ & $(4,4)$ & $\left.(\mathrm{BI})_{l, l},\left\{\alpha_{1}, \alpha_{l}\right\}\right)$ & \\
\hline
\end{tabular}

Corollary 5.1. Let $(\mathfrak{m},[g])$ be a conformal pseudo-subriemannian FGLA of semisimple type. $\operatorname{Unless}(\mathfrak{m},[g])$ is equivalent to one of $(\mathrm{H} \mathbb{C})_{p, q}(p \geqq 2, q \geqq 1),(\mathrm{H} \mathbb{C})_{1, q}(q \geqq 1),(\mathrm{H} \mathbb{H})_{p, q}(p, q \geqq 1)$, (HO), it is conformal neutral-subriemannian.

Example 5.1. Let $\mathfrak{l}=\mathfrak{l}_{-1} \oplus \mathfrak{l}_{0} \oplus \mathfrak{l}_{1}$ be a real SGLA such that $\mathfrak{l}_{-1} \neq\{0\}$. We assume that $\mathfrak{l}$ is splittable and rank $\mathfrak{l} \geqq 2$. Let $S=\bigoplus_{p<0} S_{p}$ be a faithful irreducible graded $\mathfrak{l}$-module such that $S$ is isomorphic to $\mathfrak{l}$ as an $\mathfrak{l}$-module and such that $S_{-1} \neq\{0\}$. Let $\mathfrak{t}$ be the semidirect product of $\mathfrak{l}$ by $S$. Here $S$ considers as a commutative Lie algebra. We define a gradation $\left(\mathfrak{t}_{p}\right)$ of $\mathfrak{t}$ as follows:

$$
\mathfrak{t}_{p}=\mathfrak{l}_{p} \quad(p \geqq 0), \quad \mathfrak{t}_{-1}=\mathfrak{l}_{-1} \oplus S_{-1}, \quad \mathfrak{t}_{q}=S_{q} \quad(q \leqq-2) .
$$

Then $\mathfrak{t}=\bigoplus_{p \in \mathbb{Z}} \mathfrak{t}_{p}$ becomes a GLA such that the negative part $\mathfrak{m}$ is an FGLA of the third kind. By assumption $\mathfrak{l}_{-1}$ is contragredient to $S_{-1}$ as a $\mathfrak{t}_{0}$-module. That is, there exists a $\mathfrak{t}_{0}$-module isomorphism $\varphi$ of $\mathfrak{l}_{-1}$ onto $S_{-1}^{*}$. We define a symmetric bilinear form $g$ on $\mathfrak{t}_{-1}$ as follows:

$$
g(X, Y)=g(Z, W)=0, \quad g(X, Z)=g(Z, X)=\langle\varphi(X), Z\rangle \quad\left(X, Y \in \mathfrak{l}_{-1}, Z, W \in S_{-1}\right) .
$$

Then $g$ is nondegenerate, and hence $(\mathfrak{m},[g])$ becomes a conformal neutral-subriemannian FGLA. Clearly $\mathfrak{t}$ is contained in the prolongation $\mathfrak{g}=\bigoplus_{p \in \mathbb{Z}} \mathfrak{g}_{p}$ of $(\mathfrak{m},[g])$. If $\mathfrak{g}=\bigoplus_{p \in \mathbb{Z}} \mathfrak{g}_{p}$ is of type $\left((\mathrm{BI})_{l, l},\left\{\alpha_{1}, \alpha_{l}\right\}\right)$ $(l \geqq 3)$, then $\left\{X \in \mathfrak{g}_{-1}:\left[X, \mathfrak{g}_{-2}\right]=\{0\}\right\}=\{0\}$, which is a contradiction. By Theorem 5.2, $(\mathfrak{m},[g])$ is not of semisimple type and $\mathfrak{g}_{1} \neq\{0\}$. 


\section{REFERENCES}

[1] D. V. Alekseevsky, C. Medori, and A. Tomassini, Maximally homogeneous para-CR manifolds, Ann. Glob. Anal. Geom. 30 (2006) 1-27.

[2] U. Bader and A. Nevo, Conformal actions of semisimple Lie groups on compact pseudo-Riemannian manifolds, J. Differential Geometry 60 (2002) 355-387.

[3] N. Bourbaki, Groupes et algèbres de Lie, Chapitres 4, 5 et 6, Hermann Paris, 1968.

[4] N. Bourbaki, Groupes et algèbres de Lie, Chapitres 7 et 8, Diffusion CCLS, 1975.

[5] M. G. Cowling and A. Ottazzi, Conformal maps of Carnot groups, Ann. Acad. Sci. Fennica, Mathematica 40, (2015) 203-213.

[6] A. Cap and J. Slavak, Parabolic geometries I, background and general theory, AMS, 2009.

[7] D. Djokovic, Classification of $\mathbb{Z}$-graded real semisimple Lie algebras, J. of Algebra 76, (1982) 367-382.

[8] S. Gomyo, Realization of the exceptional simple graded Lie algebras of the second kind, Algebras, groups and geometries 13, (1996) 431-464.

[9] V. V. Gorbatsevic, A. L. Onishchik, and E. B. Vinberg, Structure of Lie groups and Lie algebras, in: Lie Groups and Lie Algebras III, Encyclopedia Math. Sci. 41, Springer-Verlag Berlin, 1994.

[10] S. Kobayashi, Transformation groups in differential geometry, Springer, 1972.

[11] A. L. Onishchik, Lectures on real semisimple Lie algebras and their representations, EMS, 2004.

[12] M. Takeuchi, Cell decompositions and Morse equalities on certain symmetric spaces, J. Fac. Sci. Univ. Tokyo, 12 (1965) 81-192.

[13] N. Tanaka, On differential systems, graded Lie algebras and pseudo-groups, J. Math. Kyoto Univ. 10, (1970) $1-82$.

[14] G. Warner, Harmonic analysis on semi-simple Lie groups, I, Springer-Verlag, New York, 1972.

[15] K. Yamaguchi, Differential systems associated with simple graded Lie algebras, Advanced Studies in Pure Math. 22 (1993) 413-494.

[16] T. Yatsui, On conformal subriemannian fundamental graded Lie algebras and Cartan connections, Lobachevskii J. of Math. 36 (2015) 170-178.

MASAKAe 1-9-2, OtARU, 047-0003, JAPAN

E-mail address: yatsui@frontier.hokudai.ac.jp 